\title{
Efficient Time-Domain Finite-Difference Beam Propagation Methods for the Analysis of Slab and Circularly Symmetric Waveguides
}

\author{
Jun Shibayama, Member, OSA, Tomokazu Takahashi, Junji Yamauchi, Member, IEEE, and \\ Hisamatsu Nakano, Fellow, IEEE
}

\begin{abstract}
The generalized Douglas scheme is applied to the time-domain finite-difference beam propagation methods (TD-BPM's) in rectangular and cylindrical coordinates. High accuracy and efficiency are demonstrated through the analysis of optical pulse propagation in slab and circularly symmetric waveguides. As an example of a reflection problem, the TD-BPM in cylindrical coordinates is applied to the analysis of a fiber Bragg grating with a sinusoidal index change. Effectiveness of the present scheme is discussed in comparison with the conventional TD-BPM and the finite-difference time-domain method.
\end{abstract}

Index Terms-Fiber Bragg grating (FBG), finite-difference methods, optical beam propagation, optical waveguides, time-domain analysis.

\section{INTRODUCTION}

$\mathbf{T}$ O simulate wave propagation in an optical waveguide, we frequently use a beam propagation method (BPM) and a finite-difference time-domain method (FD-TDM). The BPM has the advantage of its high efficiency and can simulate a relatively large model. However, the BPM cannot treat reflection problems, since only the forward traveling wave is assumed. On the other hand, the FD-TDM can grasp all aspects of the optical behavior by virtue of direct discretization of Maxwell's equations, although it needs great computation resources.

Recently, to perform time-domain analysis effectively, much effort has been directed toward developing time-domain beam propagation methods (TD-BPM's) on the basis of the slowly varying envelope approximation (SVEA) [1]-[4]. Among them, the TD-BPM in which the SVEA is applied only to the time term has the advantage that reflection and diffraction can be evaluated by the BPM algorithm with slight coefficient changes [4]-[10]. With the use of the implicit scheme for discretization in time, a time step is chosen to be larger than that in the FD-TDM [4]-[6], [8]-[10].

In the FD-based TD-BPM [3]-[7], the conventional central FD scheme has been used to approximate the second derivatives in space. This results in a truncation error of $O(\Delta)^{2}$, where $\Delta$ is the sampling width in space. We should recall that, in the BPM the generalized Douglas (GD) scheme [12] has been successfully applied to the second derivatives with respect to space. The GD scheme attains a truncation error of $O(\Delta)^{4}$, provided

Manuscript received August 30, 1999.

The authors are with the College of Engineering, Hosei University, Tokyo 184-8584, Japan.

Publisher Item Identifier S 0733-8724(00)02201-5. that uniform or graded-index materials are treated [13]. This fact motivates us to apply the GD scheme to the TD-BPM [8], [10].

One should also note that the previous TD-BPM's have been formulated only in slab waveguides. From this point of view, the TD-BPM extended to circularly symmetric waveguides has begun to attract attention [11].

The purpose of this paper is to discuss the application of the GD scheme to the TD-BPM's for both rectangular and cylindrical coordinates in detail. After formulating the TD-BPM in rectangular coordinates, we demonstrate high accuracy and efficiency of the GD scheme through the analysis of pulse propagation in a straight slab waveguide. We then apply the GD scheme to the TD-BPM in cylindrical coordinates. Although the GD scheme is incorporated only into the second derivative with respect to the longitudinal direction, it still offers improvement as in the case of rectangular coordinates.

As an example of a reflection problem, we analyze a fiber Bragg grating (FBG) with a sinusoidal index change using the TD-BPM in cylindrical coordinates. The wavelength characteristics of gratings are calculated and compared with those obtained from the conventional TD-BPM [11] and the FD-TDM [14]. It is found that the sampling width in the longitudinal direction can be chosen to be four times as large as that of the conventional schemes.

\section{TD-BPM IN RECTANGULAR COORDINATES}

The wave equation to be solved is

$$
\nabla \times \nabla \times \vec{\psi}+\frac{n^{2}}{c^{2}} \frac{\partial^{2} \vec{\psi}}{\partial t^{2}}=0
$$

where $n$ is the refractive index and $c$ is the speed of light in vacuum. The dominant electric field with a centered frequency $\omega$ is formally expressed as follows:

$$
\vec{\psi}(x, t)=\vec{E}(x, t) e^{-j \omega t} .
$$

Substitution of (2) into (1) leads to

$$
\frac{n^{2}}{c^{2}} \frac{\partial^{2} \vec{E}}{\partial t^{2}}-2 j \frac{\omega n^{2}}{c^{2}} \frac{\partial \vec{E}}{\partial t}=-\nabla \times \nabla \times \vec{E}+\frac{\omega^{2} n^{2}}{c^{2}} \vec{E} .
$$

Now we adopt the SVEA such as

$$
\left|\frac{\partial^{2} \vec{E}}{\partial t^{2}}\right| \ll 2 \omega\left|\frac{\partial \vec{E}}{\partial t}\right| .
$$


With (4), (3) is reduced to the following first-order equation:

$$
-2 j \frac{\omega n^{2}}{c^{2}} \frac{\partial \vec{E}}{\partial t}=-\nabla \times \nabla \times \vec{E}+\frac{\omega^{2} n^{2}}{c^{2}} \vec{E} .
$$

We treat a two-dimensional (2-D) problem in rectangular coordinates. For TE-mode propagation, (5) can be written as follows [4]-[9]:

$$
-2 j \frac{\omega n^{2}}{c^{2}} \frac{\partial E}{\partial t}=\frac{\partial^{2} E}{\partial x^{2}}+\frac{\partial^{2} E}{\partial z^{2}}+\frac{\omega^{2} n^{2}}{c^{2}} E .
$$

Here, we discretize (6) using the alternating-direction implicit method (ADIM) as was shown in [12]. The first half-step in ADIM factorization for (6) can be separated into

$$
\begin{gathered}
\left(-2 j \frac{\omega n^{2}}{c^{2}} \frac{\partial E}{\partial t}\right)^{l}=2\left(\frac{\partial^{2} E}{\partial x^{2}}\right)^{l}+\frac{\omega^{2} n^{2}}{c^{2}} E^{l} \\
\left(-2 j \frac{\omega n^{2}}{c^{2}} \frac{\partial E}{\partial t}\right)^{l+1 / 2}=2\left(\frac{\partial^{2} E}{\partial z^{2}}\right)^{l+1 / 2}+\frac{\omega^{2} n^{2}}{c^{2}} E^{l+1 / 2} .
\end{gathered}
$$

The GD scheme can directly be applied to the second derivatives with respect to both the $x$ and $z$ directions. The next half-step is treated similarly. After a bit of algebra, we obtain the TD difference equations split into two steps:

$$
\begin{aligned}
& \eta_{x, i-1, j}^{-} E_{i-1, j}^{l+1 / 2}+\xi_{x, i, j}^{-} E_{i, j}^{l+1 / 2}+\eta_{x, i+1, j}^{-} E_{i+1, j}^{l+1 / 2} \\
& =\eta_{z, i, j-1}^{+} E_{i, j-1}^{l}+\xi_{z, i, j}^{+} E_{i, j}^{l}+\eta_{z, i, j+1}^{+} E_{i, j+1}^{l} \\
& \eta_{z, i, j-1}^{-} E_{i, j-1}^{l+1}+\xi_{z, i, j}^{-} E_{i, j}^{l+1}+\eta_{z, i, j+1}^{-} E_{i, j+1}^{l+1} \\
& \quad=\eta_{x, i-1, j}^{+} E_{i-1, j}^{l+1 / 2}+\xi_{x, i, j}^{+} E_{i, j}^{l+1 / 2}+\eta_{x, i+1, j}^{+} E_{i+1, j}^{l+1 / 2}
\end{aligned}
$$

where

$$
\begin{aligned}
& \eta_{\alpha, i, j}^{ \pm}=-\frac{j \omega n_{i, j}^{2}}{6 c^{2} \Delta t} \pm \frac{\omega^{2} n_{i, j}^{2}}{48 c^{2}} \pm \frac{1}{2 \Delta \alpha^{2}} \\
& \xi_{\alpha, i, j}^{ \pm}=-\frac{5 j \omega n_{i, j}^{2}}{3 c^{2} \Delta t} \pm \frac{5 \omega^{2} n_{i, j}^{2}}{24 c^{2}} \mp \frac{1}{\Delta \alpha^{2}}
\end{aligned}
$$

in which $\alpha=x$ or $z$. The truncation errors in (9) and (10) are reduced, when compared to the conventional formulation [4]. Since each of (9) and (10) leads to a tridiagonal matrix, the standard technique such as the Thomas algorithm can be used.

We demonstrate the efficiency of the present scheme by checking the propagation distance of an optical pulse in the slab waveguide shown in Fig. 1. The refractive indexes of the core and cladding are $n_{c o}=3.6$ and $n_{c l}=3.564$, respectively. The core width is $2 d=1.458 \mu \mathrm{m}$ and the wavelength is $\lambda=1.55 \mu \mathrm{m}$, so that only the fundamental mode propagates. The pulse wave is composed of the fundamental mode profile in the transverse direction and the Gaussian profile with a $1 / e$ full-width of $4 \mu \mathrm{m}$ in the longitudinal direction, which is generated by the one-way excitation scheme [10]. Throughout this paper, we set the absorbing boundary region at the edges of the computational window.

Fig. 2 shows the propagation distance of the peak of the optical pulse and the CPU time per propagation step. Each data

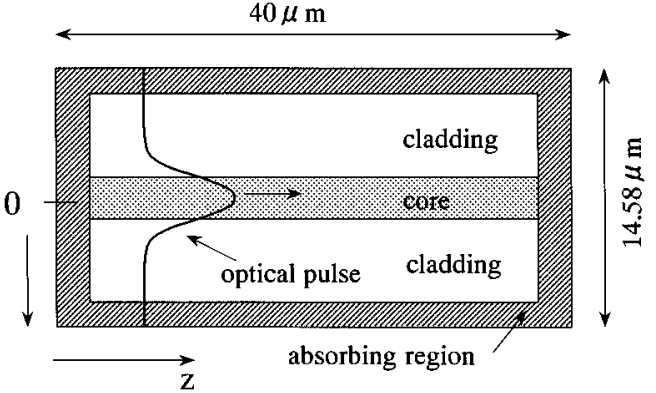

Fig. 1. Schematic of the investigated slab waveguide.

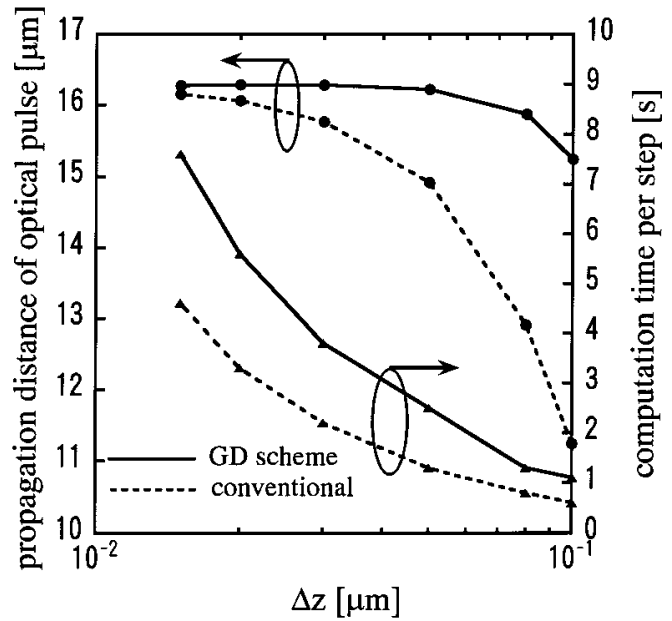

Fig. 2. Propagation distance of optical pulse and CPU time in a slab waveguide.

is expressed as a function of $\Delta z$. The propagation distance is measured at $t=200 \mathrm{fs}$. For comparison, the results obtained with the conventional central FD scheme are also shown. The calculation is carried out using a PC with 500-MHz Pentium III processor. The time step and the transverse sampling width are fixed to be $\Delta t=1 \mathrm{fs}$ and $\Delta x=d / 5=0.1458 \mu \mathrm{m}$, respectively. The number of transverse sampling points is 100 . Depending on $\Delta z$, the number of longitudinal sampling points varies from 400 to 2660 . From Fig. 2, it is seen that the propagation distance gradually converges with a decrease in $\Delta z$. For the present scheme, a converged value of $16.2 \mu \mathrm{m}$ is obtained with $\Delta z=0.05 \mu \mathrm{m}$ (note that the analytic propagation distance is calculated to be $16.7 \mu \mathrm{m}$ ). For the conventional scheme, however, it is required that $\Delta z$ be reduced to less than $0.015 \mu \mathrm{m}$ so as to obtain the same accuracy. In this case, the present scheme can reduce the CPU time to half that in the conventional scheme, while maintaining the comparable accuracy.

\section{TD-BPM IN CYLINDRICAL COORDINATES}

For circularly symmetric fields, (5) can be rewritten as follows:

$$
-2 j \frac{\omega n^{2}}{c^{2}} \frac{\partial E}{\partial t}=\frac{\partial^{2} E}{\partial r^{2}}+\frac{1}{r} \frac{\partial E}{\partial r}+\frac{\partial^{2} E}{\partial z^{2}}+\frac{\omega^{2} n^{2}}{c^{2}} E .
$$

Note that the first derivative with respect to the $r$ direction appears in (11), as contrasted with (6). In this section, we present 


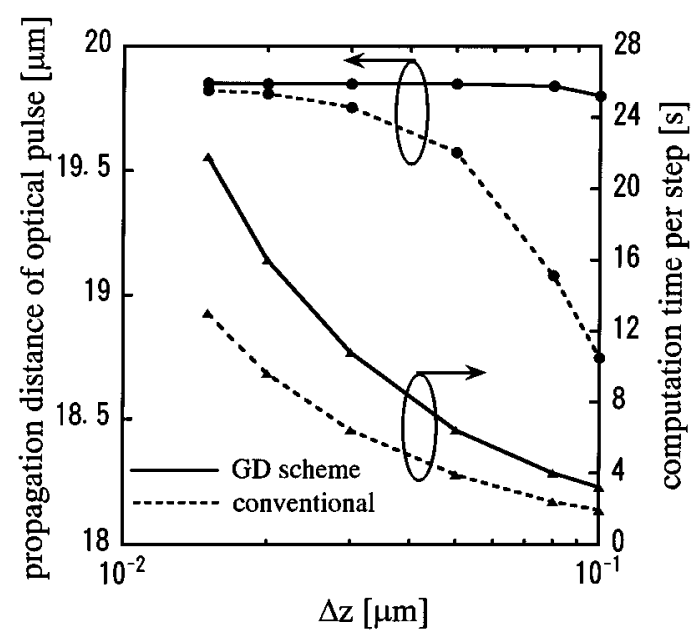

Fig. 3. Propagation distance of optical pulse and CPU time in a circularly symmetric waveguide.

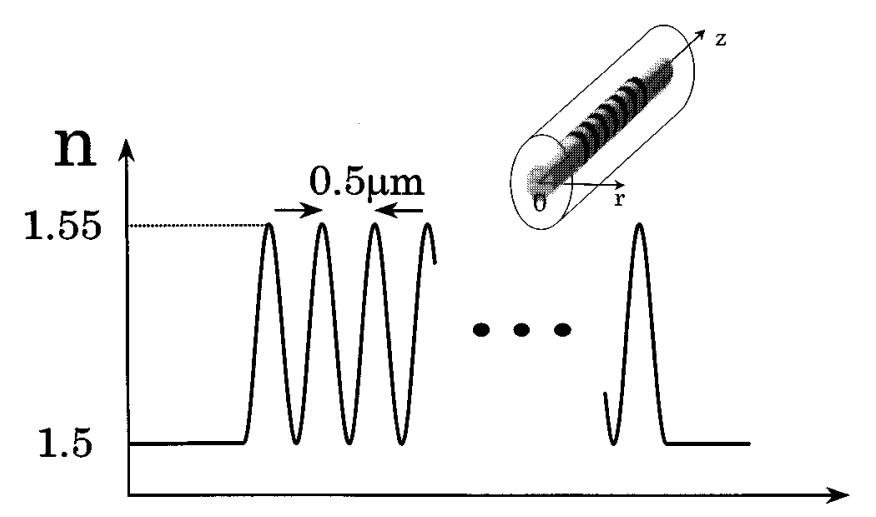

Fig. 4. Refractive index change in the core. The inset schematically illustrates the configuration of a FBG.

the detail derivation of the FD equations in cylindrical coordinates, paying attention to the treatment of the first and second derivatives in the $r$ direction.

For $r \neq 0$, the difference equation corresponding to (11) becomes

$$
\begin{aligned}
-2 j & \frac{\omega n^{2}}{c^{2}} \frac{E^{l+1}-E^{l}}{\Delta t} \\
= & \frac{\delta^{2} E^{l+1}+\delta^{2} E^{l}}{2 \Delta r^{2}}+\frac{\delta E^{l+1}+\delta E^{l}}{4 r \Delta r} \\
& +\frac{\delta^{2} E^{l+1}+\delta^{2} E^{l}}{2 \Delta z^{2}}+\frac{\omega^{2} n^{2}}{c^{2}} \frac{E^{l+1}+E^{l}}{2}
\end{aligned}
$$

where $\delta$ and $\delta^{2}$ are central difference operators for the first and second derivatives, respectively. We divide the propagation step into two steps of size $\Delta z / 2$

$$
\begin{aligned}
-2 j & \frac{\omega n^{2}}{c^{2}} \frac{E^{l+1 / 2}-E^{l}}{\Delta t / 2} \\
& =\frac{\delta^{2} E^{l+1 / 2}}{\Delta r^{2}}+\frac{\delta E^{l+1 / 2}}{2 r \Delta r}+\frac{\delta^{2} E^{l}}{\Delta z^{2}}+\frac{\omega^{2} n^{2}}{c^{2}} \frac{E^{l+1 / 2}+E^{l}}{2}
\end{aligned}
$$

and

$$
\begin{aligned}
-2 j & \frac{\omega n^{2}}{c^{2}} \frac{E^{l+1}-E^{l+1 / 2}}{\Delta t / 2} \\
= & \frac{\delta^{2} E^{l+1 / 2}}{\Delta r^{2}}+\frac{\delta E^{l+1 / 2}}{2 r \Delta r} \\
& +\frac{\delta^{2} E^{l+1}}{\Delta z^{2}}+\frac{\omega^{2} n^{2}}{c^{2}} \frac{E^{l+1}+E^{l+1 / 2}}{2} .
\end{aligned}
$$

Since the treatment regarding the two half-steps is the same, we only deal with the first half-step.

As stated in [12], (13) can be equivalently expressed as the following equations regarding the $z$ and $r$ directions:

$$
\begin{gathered}
\left(-2 j \frac{\omega n^{2}}{c^{2}} \frac{\partial E}{\partial t}\right)^{l}=2\left(\frac{\partial^{2} E}{\partial z^{2}}\right)^{l}+\frac{\omega^{2} n^{2}}{c^{2}} E^{l} \\
\left(-2 j \frac{\omega n^{2}}{c^{2}} \frac{\partial E}{\partial t}\right)^{l+1 / 2} \\
=2\left(\frac{\partial^{2} E}{\partial r^{2}}\right)^{l+1 / 2}+2\left(\frac{1}{r} \frac{\partial E}{\partial r}\right)^{l+1 / 2}+\frac{\omega^{2} n^{2}}{c^{2}} E^{l+1 / 2} .
\end{gathered}
$$

We can apply the GD scheme to the second derivative with respect to the $z$ direction in (15). It should be noted, however, that the GD scheme may not simply be applied to (16), since there exists the first derivative with respect to the $r$ direction. Fortunately, forward and backward waves traveling in the $\pm z$ directions often predominate the properties of waveguides. Therefore, improving the FD scheme in the $z$ direction offers high accuracy in the analysis of grating structures, as will be seen later.

Applying the conventional and GD schemes to the derivatives with respect to the $r$ and $z$ directions, respectively, we obtain the following FD equations:

$$
\begin{aligned}
& \eta_{r, i-1, j}^{-} E_{i-1, j}^{l+1 / 2}+\xi_{r, i, j}^{-} E_{i, j}^{l+1 / 2}+\eta_{r, i+1, j}^{-} E_{i+1, j}^{l+1 / 2} \\
& =\eta_{z, i, j-1}^{+} E_{i, j-1}^{l}+\xi_{z, i, j}^{+} E_{i, j}^{l}+\eta_{z, i, j+1}^{+} E_{i, j+1}^{l} \\
& \eta_{z, i, j-1}^{-} E_{i, j-1}^{l+1}+\xi_{z, i, j}^{-} E_{i, j}^{l+1}+\eta_{z, i, j+1}^{-} E_{i, j+1}^{l+1} \\
& \quad=\eta_{r, i-1, j}^{+} E_{i-1, j}^{l+1 / 2}+\xi_{r, i, j}^{+} E_{i, j}^{l+1 / 2}+\eta_{r, i+1, j}^{+} E_{i+1, j}^{l+1 / 2}
\end{aligned}
$$

where

$$
\begin{aligned}
\eta_{r, i-1, j}^{ \pm} & =\mp \frac{1}{4 \Delta r^{2}(i-1)} \pm \frac{1}{2 \Delta r^{2}} \\
\eta_{r, i+1, j}^{ \pm} & = \pm \frac{1}{4 \Delta r^{2}(i-1)} \pm \frac{1}{2 \Delta r^{2}} \\
\xi_{r, i, j}^{ \pm} & =-\frac{2 j \omega n_{i, j}^{2}}{c^{2} \Delta t} \pm \frac{\omega^{2} n_{i, j}^{2}}{4 c^{2}} \mp \frac{1}{\Delta r^{2}} \\
\eta_{z, i, j}^{ \pm} & =-\frac{j \omega n_{i, j}^{2}}{6 c^{2} \Delta t} \pm \frac{\omega^{2} n_{i, j}^{2}}{48 c^{2}} \pm \frac{1}{2 \Delta z^{2}} \\
\xi_{z, i, j}^{ \pm} & =-\frac{5 j \omega n_{i, j}^{2}}{3 c^{2} \Delta t} \pm \frac{5 \omega^{2} n_{i, j}^{2}}{24 c^{2}} \mp \frac{1}{\Delta z^{2}} .
\end{aligned}
$$


On the fiber axis, $r=0$, L'Hospital's rule is applied to the second term in the right-hand side of (11). Then, we obtain

$$
-2 j \frac{\omega n^{2}}{c^{2}} \frac{\partial E}{\partial t}=2 \frac{\partial^{2} E}{\partial r^{2}}+\frac{\partial^{2} E}{\partial z^{2}}+\frac{\omega^{2} n^{2}}{c^{2}} E .
$$

One may notice that the GD scheme can also be introduced into the $r$ direction in (19). However, in order to match the resultant equations for $r=0$ with those for $r \neq 0$, we apply the GD scheme only to the $z$ direction. As a result, the FD equations for $r=0$ are as follows:

$$
\begin{aligned}
& -2 / \Delta r^{2} E_{0, j}^{l+1 / 2}+\xi_{r, 1, j}^{-} E_{1, j}^{l+1 / 2} \\
& =\eta_{z, 1, j-1}^{+} E_{1, j-1}^{l}+\xi_{z, 1, j}^{+} E_{1, j}^{l}+\eta_{z, 1, j+1}^{+} E_{1, j+1}^{l} \\
& \eta_{z, 1, j-1}^{-} E_{1, j-1}^{l+1}+\xi_{z, 1, j}^{-} E_{1, j}^{l+1}+\eta_{z, 1, j+1}^{-} E_{1, j+1}^{l+1} \\
& =2 / \Delta r^{2} E_{2, j}^{l+1 / 2}+\xi_{r, 1, j}^{+} E_{1, j}^{l+1 / 2}
\end{aligned}
$$

where

$$
\begin{aligned}
& \xi_{r, 1, j}^{ \pm}=-\frac{2 j \omega n_{1, j}^{2}}{c^{2} \Delta t} \pm \frac{\omega^{2} n_{1, j}^{2}}{4 c^{2}} \mp \frac{2}{\Delta r^{2}} \\
& \eta_{z, 1, j}^{ \pm}=-\frac{j \omega n_{1, j}^{2}}{6 c^{2} \Delta t} \pm \frac{\omega^{2} n_{1, j}^{2}}{48 c^{2}} \pm \frac{1}{2 \Delta z^{2}} \\
& \xi_{z, 1, j}^{ \pm}=-\frac{5 j \omega n_{1, j}^{2}}{3 c^{2} \Delta t} \pm \frac{5 \omega^{2} n_{1, j}^{2}}{24 c^{2}} \mp \frac{1}{\Delta z^{2}} .
\end{aligned}
$$

We can again find that each of (17), (18), (20) and (21) is a tridiagnal form, allowing the use of the Thomas algorithm.

For the assessment of the present scheme, we analyze an optical fiber whose refractive indexes of the core and cladding are $n_{c o}=1.50$ and $n_{c l}=1.45$, respectively. The core radius is $r=1.0 \mu \mathrm{m}$. The pulse wave to be used is the same as that used in Fig. 2 except for the $\mathrm{LP}_{01}$ mode profile in the transverse direction. The wavelength is $\lambda=1.55 \mu \mathrm{m}$.

Fig. 3 shows the propagation distance observed at $t=100$ fs and the CPU time per propagation step, in which the data expressed as a function of $\Delta z$ are obtained by the same $\mathrm{PC}$ as that used in Fig. 2. Due to the symmetry of the fiber, only the half section is analyzed. The time step and the transverse sampling width are fixed to be $\Delta t=1 \mathrm{fs}$ and $\Delta r=r / 19.5 \simeq 0.05$ $\mu \mathrm{m}$, respectively. The number of transverse sampling points is 300. Depending on $\Delta z$, the number of longitudinal sampling points varies from 420 to 2800 . It is seen that the result obtained from the present scheme almost converges at $\Delta z=0.05 \mu \mathrm{m}$. A converged value of $19.85 \mu \mathrm{m}$ agrees well with an analytic solution of $20.0 \mu \mathrm{m}$. In contrast, the result obtained from the conventional scheme converges for $\Delta z$ of less than $0.015 \mu \mathrm{m}$. Eventually, the present scheme provides improved accuracy as observed for rectangular coordinates in Fig. 2, although the GD scheme is applied only to the $z$ direction.

\section{ANALYSIS OF A FIBER BRAGg GRATING}

To demonstrate the validity of the present scheme for a reflection problem, we analyze a FBG with a sinusoidal index change using the TD-BPM in cylindrical coordinates. The refractive indexes in the core changes between 1.50 and 1.55 as shown in

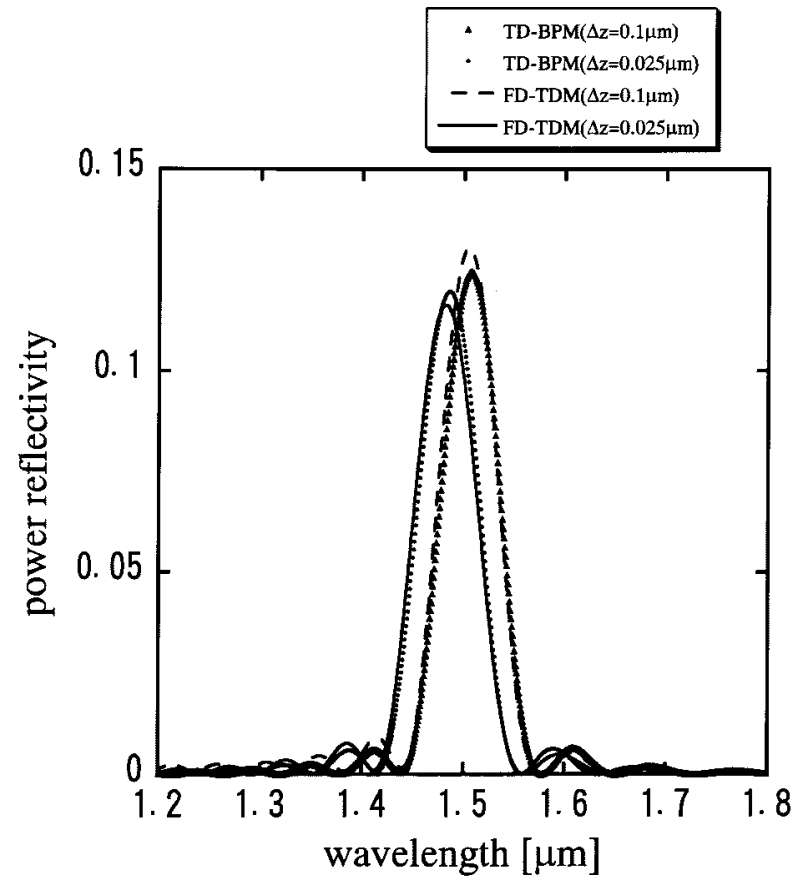

(a)

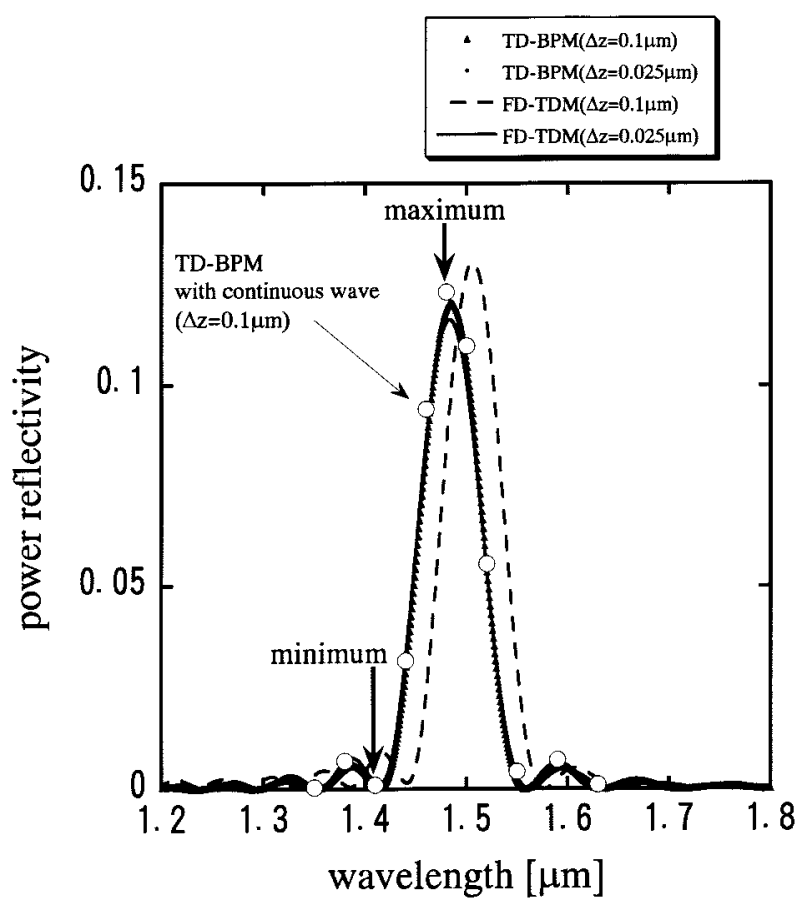

(b)

Fig. 5. Power reflectivity. (a) Conventional scheme and (b) GD scheme.

Fig. 4, while that of the cladding is fixed to be $n_{c l}=1.45$. The grating period is $0.5 \mu \mathrm{m}$ and there are 20 periods in the core. The core diameter is $2 R=2 \mu \mathrm{m}$. The incident field is the same pulse wave as that used in Fig. 3. The spectrum of the power reflectivity is calculated using the discrete Fourier transform.

Fig. 5 shows the power reflectivity for a parameter of $\Delta z$, in which the results obtained from the FD-TDM [14] are also included for reference. The conventional and GD schemes are employed for the TD-BPM in Fig. 5(a) and (b), respectively. 


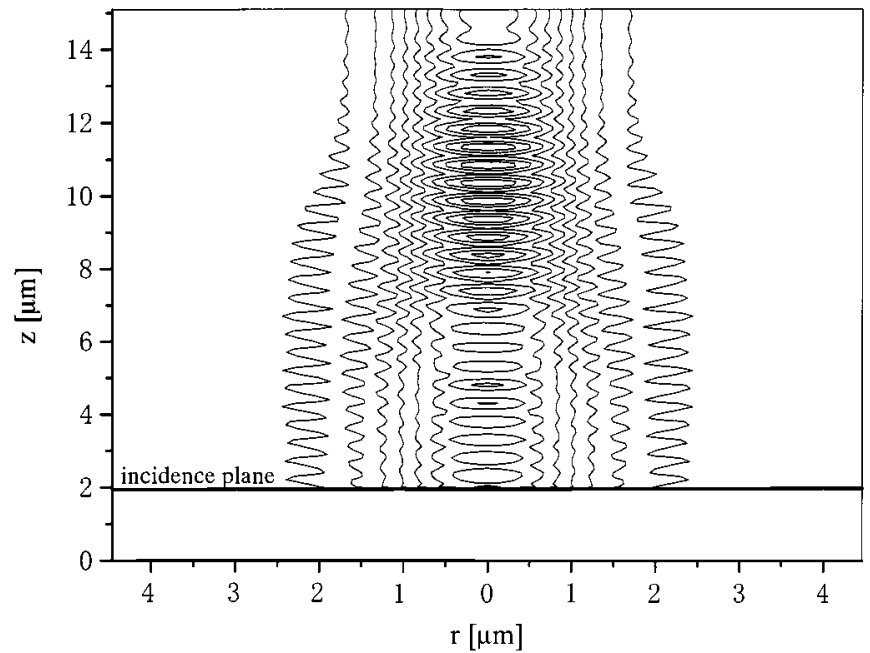

(a)

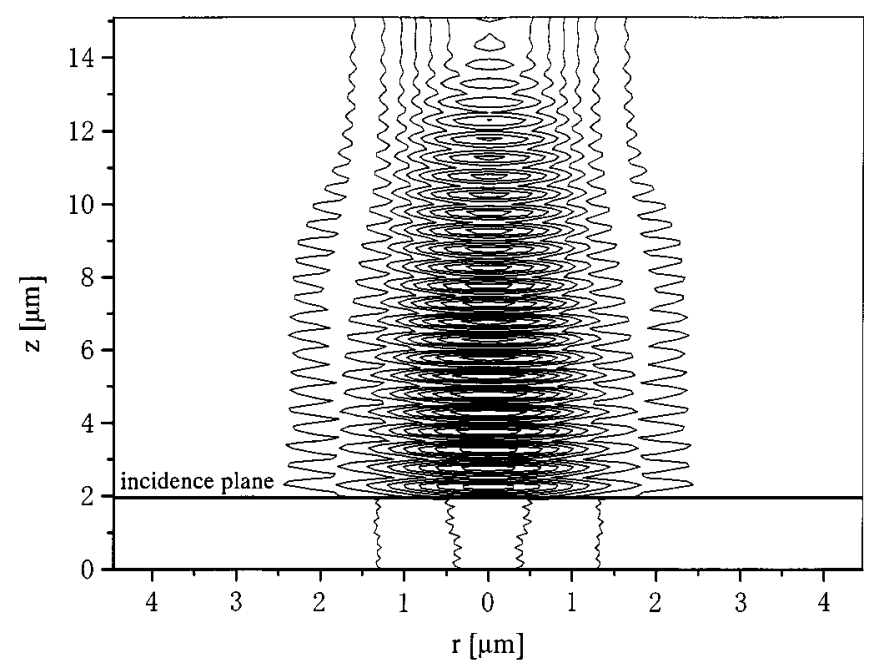

(b)

Fig. 6. Field contour plots observed for (a) minimum reflectivity and (b) maximum reflectivity.

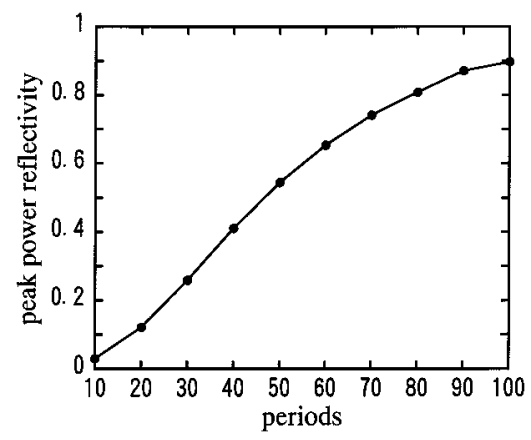

Fig. 7. Peak power reflectivity as a function of the number of grating periods.

The numerical parameters are fixed as follows: $\Delta t=2 \mathrm{fs}$ and $\Delta r=R / 19.5$. In Fig. 5(a), the peak wavelength obtained by the conventional scheme almost agrees with that obtained by the FD-TDM, provided that the same sampling widths in the $z$ direction are used. This is due to the fact that the truncation error of the conventional scheme is identical with that of the FD-TDM, i.e., $O(\Delta z)^{2}$. For these two schemes, the sampling width should be reduced to be $0.025 \mu \mathrm{m}$ so as to obtain a converged result (in this case, $\Delta t$ for the FD-TDM is $0.11 \mathrm{fs}$ ). In contrast, Fig. 5(b) shows that the GD scheme yields a converged result for $\Delta z=0.1 \mu \mathrm{m}$ (the result is superimposed on that for $\Delta z=0.025 \mu \mathrm{m}$ ), which compares favorably with that obtained by the FD-TDM for $\Delta z=0.025 \mu \mathrm{m}$. The CPU time in the present TD-BPM is reduced to $25 \%$ of that in the FD-TDM. The use of a lager $\Delta z$ contributes to substantial reduction in CPU time.

We also calculate the power reflectivity using the present TD-BPM with the one-way continuous-wave (CW) excitation scheme [10], which is plotted in Fig. 5(b). The longitudinal sampling width is chosen to be $\Delta z=0.1 \mu \mathrm{m}$. Good agreement is found between the results for the continuous wave and the pulse wave excitations.

A one-way CW excitation gives a steady-state field in which the reflected and total fields are clearly separated [14], [10]. Fig. 6(a) and (b) illustrates the field distributions observed for minimum and maximum reflectivities, respectively [the data are obtained at the points marked in Fig. 5(b)]. For both cases, the field exhibits a complicated standing wave, due to the multiple reflection from gratings. In the former, the reflected wave cannot be seen in the reflected field region, since it is canceled out. In the latter, the peak position of the standing wave is found to be close to the input side of the grating.

So far, the number of grating periods has been fixed to be 20 , just for the assessment of the present scheme. Finally, we investigate the effect of the number of grating periods on the peak power reflectivity. As expected, the reflectivity increases, as the number of grating periods is increased. As seen in Fig. 7 , the power reflectivity reaches approximately $90 \%$ for 100 periods.

\section{CONCLUSION}

Efficient TD-BPM's for the analysis of slab and circularly symmetric waveguides have been formulated using the GD scheme. To demonstrate the efficiency, we calculate the propagation distance of optical pulse in straight waveguides. Numerical results show substantial improvement in the present TD-BPM's both for rectangular and cylindrical coordinates. As an example of a reflection problem, a FBG with a sinusoidal index change is analyzed by the TD-BPM in cylindrical coordinates. It is shown that a sampling width can be chosen to be four times as large as those of the conventional TD-BPM and the FD-TDM, maintaining comparable accuracy. The CPU time in the present TD-BPM is reduced to $25 \%$ of that in the FD-TDM.

\section{REFERENCES}

[1] W. P. Huang, C. L. Xu, and J. Chrostowski, "A time-domain propagating scheme for simulation of dynamics of optical guided-wave devices," IEEE Photon. Technol. Lett., vol. 5, pp. 1071-1073, 1993.

[2] M. Scalora and M. E. Crenshaw, "A beam propagation method that handles reflections," Opt. Commun., vol. 108, pp. 191-196, 1994.

[3] R. Y. Chan and J. M. Liu, "Time-domain wave propagation in optical structures," IEEE Photon. Technol. Lett., vol. 6, pp. 1001-1003, 1994.

[4] P. L. Liu, Q. Zhao, and F. S. Choa, "Slow-wave finite-difference beam propagation method," IEEE Photon. Technol. Lett., vol. 7, pp. 890-892, 1995. 
[5] Y. Yuan, R. Jambunathan, J. Singh, and P. Bhattacharya, "Finite-difference time-domain analysis and experimental examination of the performance of a coupled-cavity MQW laser/active waveguide at $1.54 \mu \mathrm{m}$," IEEE J. Quantum Electron., vol. 33, pp. 408-415, 1997.

[6] G. H. Jin, J. Harari, J. P. Vilcot, and D. Decoster, "An improved timedomain beam propagation method for integrated optics components," IEEE Photon. Technol. Lett., vol. 9, pp. 348-350, 1997.

[7] F. Ma, "Slowly varying envelope simulation of optical waves in time domain with transparent and absorbing boundary conditions," J. Lightwave Technol., vol. 15, pp. 1974-1985, 1997.

[8] J. Shibayama, J. Yamauchi, and H. Nakano, "Formulation of a time-domain finite-difference beam propagation method based on the generalized Douglas scheme," IEICE Trans. C-1, vol. 81, pp. 38-39, 1998.

[9] M. Hikari, M. Koshiba, and Y. Tsuji, "A time domain beam propagation method based on a finite element scheme," IEICE Trans. C-1, vol. 81, pp. 667-673, 1998 .

[10] J. Yamauchi, J. Shibayama, and H. Nakano, "Application of the generalized Douglas scheme to optical waveguide analysis," Opt. Quantum Electron., vol. 31, pp. 675-687, 1999.

[11] J. Shibayama, T. Takahashi, J. Yamauchi, and H. Nakano, "Extended time-domain finite-difference beam propagation methods,", Integrated Photonics Research, RTuI2, Santa Barbara, 1999.

[12] J. Yamauchi, J. Shibayama, O. Saito, O. Uchiyama, and H. Nakano, "Improved finite-difference beam-propagation method based on the generalized Douglas scheme and its application to semivectorial analysis," $J$. Lightwave Technol., vol. 14, pp. 2401-2406, 1996.

[13] J. Shibayama, K. Matsubara, M. Sekiguchi, J. Yamauchi, and H. Nakano, "Efficient nonuniform schemes for paraxial and wide-angle finite-difference beam propagation methods," J. Lightwave Technol., vol. 17, pp. 677-683, 1999 .

[14] J. Yamauchi, M. Nibe, and H. Nakano, "Scalar FD-TD method for circularly symmetric waveguides," Opt. Quantum Electron., vol. 29, pp. 451-460, 1997

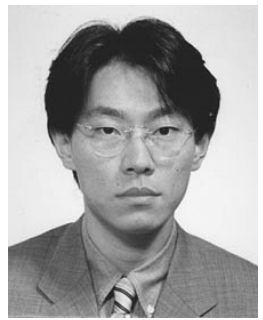

Jun Shibayama was born in Kashiwa, Chiba, Japan, on July 1, 1969. He received the B.E. and M.E. degrees from Hosei University, Tokyo, Japan, in 1993 and 1995 , respectively.

In 1995, he joined Opto-Technology Laboratory, Furukawa Electric Company, Ltd., Ichihara, Chiba, Japan. Since 1999, he has been an Assistant of Hosei University. His research interests include numerical analysis of optical waveguides.

Mr. Shibayama is a member of the Optical Society of America (OSA) and the Institute of Electronics, Information and Communication Engineers (IEICE) of Japan.

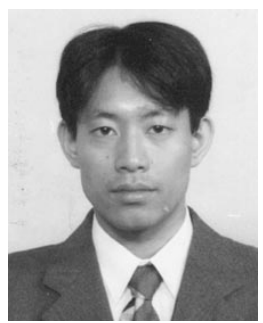

Tomokazu Takahashi was born in Tokyo,Japan, on March 2, 1977. He received the B.E. degrees from Hosei University, Tokyo, Japan, in 1999 and is currently pursuing the M.E. degree.

Mr. Takahashi is a member of the Institute of Electronics, Information and Communication Engineers (IEICE) of Japan.

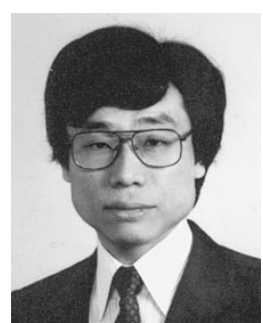

Junji Yamauchi (M'85) was born in Nagoya, Japan, on August 23, 1953. He received the B.E., M.E., and Dr.E. degrees from Hosei University, Tokyo, Japan, in 1976, 1978, and 1982, respectively.

From 1984 to 1988 , he served as a Lecturer at the Electrical Engineering Department of Tokyo Metropolitan Technical College. Since 1988, he has been a member of the Faculty of Hosei University, where he is now a Professor of Electric Informatics. His research interests include optical waveguides and circularly polarized antennas.

Dr. Yamauchi is a member of the Optical Society of America (OSA) and the Institute of Electronics, Information and Communication Engineers (IEICE) of Japan.

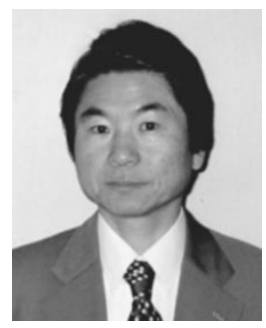

Hisamastu Nakano (M'75-SM'87-F'92) was born in Ibaraki, Japan, on April 13,1945. He received the B.E., M.E., and Dr.E. degrees in electrical engineering from Hosei University, Tokyo, in 1968, 1970, and 1974, respectively.

Since 1973, he has been a member of the Faculty of Hosei University, where he is now a Professor of Electric Informatics. His research topics include numerical methods for antennas, electromagnetic wave scattering problems, and lightwave problems. He has published more than 130 refereed journal papers and 90 international symposium papers on antenna and relevant problems. He is the author of the book Helical and Spiral Antennas (New York: Wiley Research, 1987). In 1996, he published the chapter "Antenna Analysis Using Integral Equations," in the book Analysis Methods of Electromagnetic Wave Problems (Dedham, MA: Artech House, vol. 2). He has developed a parabolic antenna using backfire helical feed for direct reception of broadcasting satellite TV programs (DBS). He has also developed two types of small indoor flat DBS antennas using novel elements: curled and extremely low-profile helical elements. His other developments include microstrip antennas for global positioning system (GPS), personal handy telephone antennas, and small dual-polarization Cassegrain antennas for direct reception of communication satellite TV programs. He was a Visiting Associate Professor at Syracuse University, Syracuse, NY (May to September, 1981), where he worked on numerical analysis of electromagnetic coupling between wires and slots, a Visiting Professor at University of Manitoba, Canada (from March to September, 1986), researching numerical techniques for analysis of microstrip antennas, and a Visiting Professor at University of California, Los Angeles (from September 1986 to March 1987), working on microstrip line antenna analysis.

Dr. Nakano was the recipient of an International Scientific Exchange Award from the Natural Sciences and Engineering Research Council of Canada. In 1987, he received the Best Paper Award of the IEE International Conference on Antenna and Propagation. In 1994, he received the IEEE AP-S Best Application Paper Award (H. A. Wheeler Award). He is an Associate Editor of IEEE ANTENNAS AND PROPAGATION MAGAZINE. 